\title{
Making Money out of Publicly Available
}

\section{Information}

\author{
Alan D. Morrison* \\ Saïd Business School and Merton College, \\ University of Oxford \\ Nir Vulkan \\ Saïd Business School and Worcester College, \\ University of Oxford
}

This version: July 2004

${ }^{*}$ We thank Pete Kyle, Tarun Ramadorai, Jos van Bommel and seminar participants at Nuffield College and the Saïd Business School for useful discussions of the ideas in this paper.

Correspondence address: Alan Morrison, Merton College, Oxford, OX1 4JD, U.K. Telephone: (+44) 1865276 343. Fax: (+44) 1865276 361. email: alan.morrison@sbs.ox.ac.uk 


\title{
Making Money out of Publicly Available Information
}

\begin{abstract}
It is received financial wisdom that when there is free entry by speculators, it is impossible to generate net profits on publicly available information. In this paper we study a version of the standard Kyle (85) model with endogenous information acquisition and we find that equilibria exist with free entry in which speculators make positive profits. Moreover, these equilibria are robust.
\end{abstract}

Keywords: Market maker model, beliefs, information accquisition.

JEL Classification: D82, G14.

\section{Introduction}

It is widely believed that it is impossible to make profits from trading on publicly available information. This belief is supported by the efficient markets hypothesis, which states that information will be correctly reflected by market prices. Early work by Grossman and Stiglitz (1980) demonstrates that competitive agents with gather information until the net trading profit from becoming informed is zero. A substantial literature has subsequently arisen which models the process by which information is reflected in prices when speculators behave strategically. The seminal paper in this field was written by Kyle (1985), in which information endowments are exogenous. Endogenous information acquisition has been examined in a number of related papers. ${ }^{1}$ A common feature of these is that with free entry, speculator profits are precisely equal to the cost of the information which they use.

In this paper we study a version of Kyle's (1985) model with free entry. Like Kyle, we constrain prices to be informationally efficient. We explicitly model the decisions of speculators whether or

\footnotetext{
${ }^{1}$ Verrecchia (1982) examines information acquisition by risk averse competitive traders; Kyle (1989) models information acquisition in a limit order model of strategic traders; endogenous information acquisition with free entry by speculators is examined in Kyle's (1985) framework by amongst others Admati and Pfleiderer (1988), Spiegel and Subrahmanyam (1992) and Foster and Viswanathan (1993). Morrison (2003) considers a model without free entry but with information acquisition, where market makers select endogenously the precision of their signal.
} 
not to acquire information. We show that equilibria exist in which speculators make positive profits after paying for their information, and that these equilibria are robust.

Why do positive expected profits not attract entry from additional speculators who compete the profits down to zero? In models with endogenous information acquisition beliefs off the equilibrium path can have a dramatic effect on the profits which speculators make on the equilibrium path. In particular, if speculators already in the market interpret off equilibrium path entry by additional speculators as noise trading then they will respond by trading more aggressively on the information which they have. This aggression, coupled with the activities of the additional speculator, will render prices so informative that the additional speculator makes an expected loss. In equilibrium potential new speculators will anticipate this effect and so will choose not to acquire information.

The intuition that beliefs can serve as a barrier to entry is consistent with statements by successful speculators. For example, the renowned hedge fund manager Paul Tudor Jones stated in a 1989 interview that he did not rely upon privileged information to generate his profits:

[...] the perception is going to be that we did well as a trading firm, while other people were hurt, because we had some knowledge. It is not that we had any [...] knowledge that other people did not have [...] (Schwager, 1989, p.137: this theme is developed in numerous interviews throughout the book).

The rest of the paper is organized in the following way. Section 2 sets up the model and defines an equilibrium. In section 3 we prove the existence of equilibria in which speculators make positive profits. In section 4 we examine the comparative statics of the model and show that the equilibrium in which speculators make positive profits is robust. Section 5 concludes.

\section{The Model}

We consider a market for a single risky security, whose value $S$ is a random variable:

$$
\tilde{S}=\bar{S}+\tilde{\alpha}
$$

where the expected value $\bar{S}$ of the security is common knowledge and $\tilde{\alpha}$ is a randomly distributed noise term with mean 0 and variance $V_{\alpha} \cdot{ }^{2}$ There are three types of agents in the model: a risk neutral competitive market maker, $\tilde{m}$ liquidity traders, and $n$ informed traders.

\footnotetext{
${ }^{2}$ Throughout the paper, random variables are indicated by a tilde $(\tilde{X})$ : realisations of the variables are shown unadorned $(X)$.
} 
The trade sizes $\tilde{y}_{1}, \tilde{y}_{2}, \ldots, \tilde{y}_{\tilde{m}}$ of the liquidity traders are determined exogenously as independent draws from a normal distribution with mean 0 and variance $V_{L} V_{\alpha}$. For every $i, \tilde{y}_{i}$ is independent of $\tilde{\alpha}$.

The informed traders have access to a costly information-gathering technology which gives each of them the same imperfect signal of

$$
\tilde{\alpha}+\tilde{\varepsilon} \sqrt{V_{\alpha}}
$$

where $\tilde{\varepsilon}$ is a draw from $N(0,1)$ which is the same for every trader, and $\frac{1}{V_{\alpha}}$ is the precision of the signal.

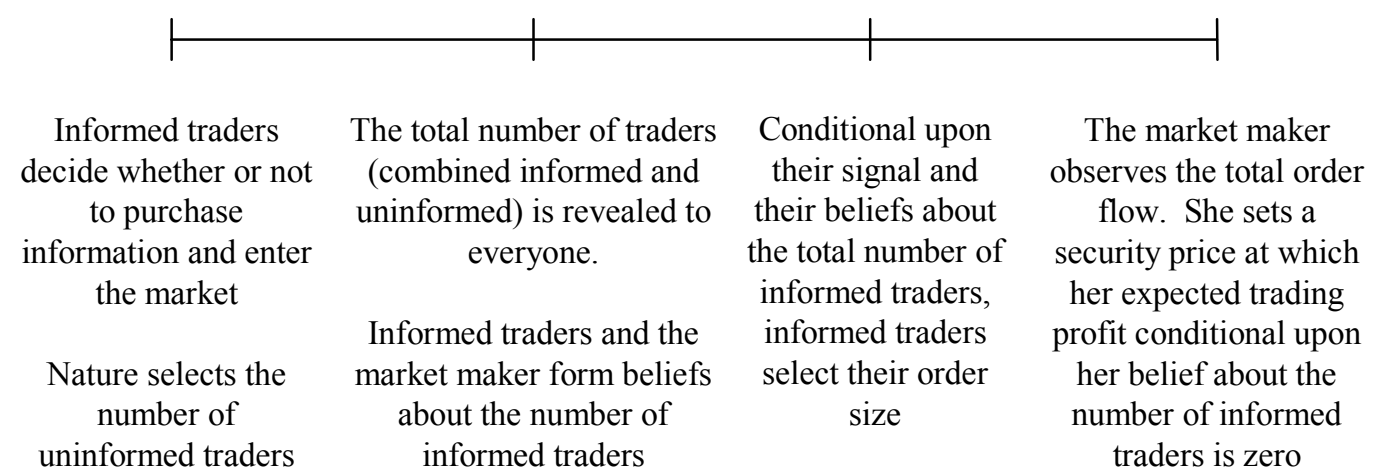

Figure 1: Timeline for the information acquisition and trading game.

The operation of the market is illustrated in figure 1. Firstly, the informed traders decide whether or not to enter the market and to purchase information and nature selects the number $\tilde{m}$ of uninformed traders from a probability distribution with support $[1, \infty)$.

The total number $n+m$ of traders is revealed to the market. While this clearly a simplification, we believe that the assumption is justified. Disclosure requirements around the world require stockholders to reveal their existence. For example, in the U.S., 13-F filings of the SEC reveal on a quarterly basis the number of managers holding a significant position in each stock. In the U.K. the London Stock Exchange requires that all stock holdings in excess of $3 \%$ be disclosed immediately. ${ }^{3}$ The assumption is also consonant with anecdotal evidence gleaned from our conversations with traders who believe they know the approximate size of the marketplace.

The informed traders and the market market form beliefs for the values of $m$ and $n$. Conditional upon their signals and their beliefs about $m$ and $n$ the informed traders select trade sizes $\tilde{x}_{1}, \tilde{x}_{2}$,

\footnotetext{
${ }^{3}$ Similar disclosure rules obtain in Italy, Norway, the Netherlands, Canada, Switzerland, Sweden, Finland, Denmark, France, Spain and Germany.
} 
$\ldots, \tilde{x}_{n}$

Denote by

$$
\tilde{\omega} \equiv \sum_{i=1}^{n} \tilde{x}_{i}+\sum_{j=1}^{\tilde{m}} \tilde{y}_{j}
$$

the total order flow which the market maker observes. She is competitive and sets a price at which her expected profit conditional upon $\tilde{\omega}$ and her beliefs about $m$ and $n$ is zero.

We search for equilibria which are linear and symmetric:

Definition 1 A symmetric linear Nash equilibrium for this game is one in which:

1. The market maker's pricing strategy is:

(a) Competitive: $E\{\tilde{P} \mid \tilde{\omega}\}=0$;

(b) Linear: $\tilde{P}=\bar{S}+\lambda \tilde{\omega}$.

2. The informed traders' strategies are linear: informed trader order size has a linear dependence upon the received signal:

$$
x=\beta\left(\tilde{\alpha}+\tilde{\varepsilon} \sqrt{V_{\alpha}}\right) .
$$

3. The beliefs which informed traders form about $m$ and $n$ are correct.

4. Informed traders' actions maximize their expected income, given the actions of other informed traders.

The model which we describe here is almost identical to the one which Kyle examines in his classic 1985 paper, extended to provide for endogenous information acquisition and free entry of informed traders. Other papers have allowed informed trader entry, but they have assumed that the precise number of informed traders is common knowledge, and hence that entry will occur until the expected profit from information acquisition is equal to zero. Our model extends this literature by making the reasonable assumption that,although traders will be aware of increased order flow in a market, they typically be unable to distinguish between informed and uninformed orders. We are therefore able to consider the process by which expectations about information acquisition are formed, and the equilibrium consequences of this process. 
3. Solving the Model

In this section we show that there will typically be multiple symmetric linear Nash equilibria of the game outlined in section 2, each of which will be supported by rational beliefs about the respective numbers $m$ and $n$ of liquidity and informed traders. One of these is the zero expected profit equilibrium identified at the end of section 2: the equilibrium considered in the existing literature is therefore a special case of the model which we present.

We define a belief function to be a mapping $\mathbb{N} \rightarrow \mathbb{N}$ which assigns to any observed number $(m+n)$ of traders a number $n$ of informed traders. In equilibrium the market maker and every speculator will have the correct beliefs and hence each will have the same belief function. Since when they make their entry decisions, the speculators do not know the number $m$ of liquidity traders, the number $n$ of speculators cannot depend upon $m$. In other words, in equilibrium the belief function must assume a constant value: $N \mapsto n .^{4}$ As a result, in equilibrium both the market maker and the speculators will attribute a observed number of traders to a high value for $m$.

Lemma 2 Suppose that $n$ speculators gather information, that they use the belief function $N \mapsto$ $n$ to determine their optimal order size, and that the market maker uses the same function to determine the price of the security. An $(n+1)$ th speculator will generate the following expected profit, excluding the cost $c$ of information production:

$$
\Pi(n) \equiv \frac{V_{\alpha}}{4 E\left\{\frac{1}{\sqrt{1+\tilde{m}}}\right\}(n+1)} \sqrt{\frac{V_{L}}{2 n}} .
$$

Lemma 2 demonstrates that, when $\Pi(n)<c$, the belief function $N \mapsto n$ can, if held by $n$ speculators and the market maker, serve to deter entry by an $(n+1)$ th speculator. This belief function will be rational if each of the $n$ speculators who holds it makes a profit in expectation. Lemma 3 establishes conditions under which this is possible.

Lemma 3 Suppose that $n$ speculators gather information, that they and the market maker use the belief function $N \mapsto n$ to determine optimal order size, and that $\Pi(n)<c$. Then, excluding the cost $c$ of information production each speculator will earn the following expected profit:

$$
P(n) \equiv \frac{V_{\alpha} E\{\sqrt{\tilde{m}}\}}{n+1} \sqrt{\frac{V_{L}}{2 n}}
$$

\footnotetext{
${ }^{4}$ Note that in our model, fewer than $n$ traders will never be observed in equilibrium. For completeness, we can assume that the belief function for fewer than $n$ observed traders is the identity mapping.
} 
We combine lemmas 2 and 3 to obtain necessary and sufficient conditions for the existence of symmetric linear equilibria to for our model:

\section{Proposition 4}

1. Incumbent speculators always have a larger expected profit than new entrants:

$$
P(n)>P(n+1)>\Pi(n)
$$

2. A symmetric linear Nash equilibrium can be supported by the belief function $N \mapsto n$ whenever condition 4 is satisfied:

$$
P(n)>c>\Pi(n) .
$$

Let $s^{Z P}$ and $s^{M P}$ solve equations 5 and 6 :

$$
\begin{aligned}
& P\left(s^{Z P}\right)=c \\
& \Pi\left(s^{M P}\right)=c
\end{aligned}
$$

and define

$$
\begin{aligned}
n^{Z P} & \equiv \operatorname{int}\left(s^{Z P}\right) \\
n^{M P} & \equiv \operatorname{int}\left(s^{M P}\right)+1 .
\end{aligned}
$$

\section{Lemma 5}

1. $\Pi\left(n^{Z P}\right)<c$;

2. $n^{M P} \leq n^{Z P}$.

Proof. For the first part, note that by definition, $P\left(n^{Z P}+1\right)<c$ and that, by equation 3, $P\left(n^{Z P}+1\right)>\Pi\left(n^{Z P}\right)$. Since $\Pi\left(n^{Z P}\right)<c$ we must have $n^{Z P}>s^{M P}$, whence the second part follows immediately.

Since $P^{\prime}(n)<0, n^{Z P}$ is the highest number of speculators which can coexist, and by part 1 of lemma 5 the belief function $N \mapsto n^{Z P}$ supports an equilibrium with $n^{Z P}$ speculators. This corresponds to the standard story in which free entry by speculators drives the profit per speculator down to zero (or as close to it as possible, given the requirement that that there be an integer number of speculators). Our model therefore includes the equilibria considered in other models with free entry by speculators.

Note that $\Pi\left(n^{M P}\right)<0$ (because $\Pi^{\prime}(n)<0$ ) and hence by part 2 of lemma 5 the belief function $N \mapsto n^{M P}$ supports an equilibrium with $n^{M P}$ speculators. Since $\Pi\left(n^{M P}-1\right) \geq 0, n^{M P}$ 
is the smallest number of speculators which can exist in an equilibrium and, since $P^{\prime}(n)<0$, it corresponds to the largest per-speculator profit.

In particular note that when $n^{M P}$ is strictly less than $n^{Z P}$, our model supports a range of equilibria in some of which (those with fewer than $n^{Z P}$ speculators) each speculator makes a nonzero profit. We demonstrate by example in section 4 that there exist parameter values for which this is the case. By carefully modelling the information acquisition process, we have therefore expanded the set of equilibria beyond those previously considered.

In other words, equilibria exist in which even with free entry fewer than the maximum possible number of speculators $n^{Z P}$ enter. In these equilibria each speculator makes a non-zero profit. The intuition for this result is simple. If in equilibrium the $n$ incumbent speculators all believe that they are the only speculators in the market then they assume that with $N$ traders, $N-n$ are liquidity traders. They will therefore assume that a higher than expected total number of traders $N$ has been caused by additional liquidity traders. Since the additional liquidity traders serve to disguise the actions of informed traders the incumbent speculators will respond to them by trading more aggressively. If an $(n+1)$ th speculator elects to enter the market he will therefore face an aggressive response from the existing traders. This will render the price more informative and so will reduce his expected profits from entry below the cost of information. The beliefs of the $n$ speculators are therefore rational in equilibrium.

In fact, we can show that our results still hold if we refine the solution concept to sequential equilibrium (Kreps and Wilson, 1982). To do so, note that the equilibrium beliefs in our model with $n$ speculators are the limit as $\varepsilon$ tends to zero of completely mixed beliefs in which all traders assume that the type distribution of $N$ observed traders is the sum of $\min (N, n)$ Bernoulli variables which are speculators with probability $1-\varepsilon$ and uninformed with probability $\varepsilon$; and $N-\min (M, n)$ Bernoulli variables which are speculators with probability $\varepsilon$ and uninformed with probability $1-\varepsilon$. Given these beliefs at least $n$ speculators will enter. Entry and order submission strategies are continuous functions of $\varepsilon$ and in the limit as $\varepsilon$ tends to zero, they converge to those described in proposition 4 . 


\section{Comparative Statics}

We define the percentage return which a trader receives to be $\frac{E\{\text { Profit }\}-\text { Information Cost }}{\text { Information Cost }}$, and let $k=$ $n^{Z P}-n^{M P}$ be the difference between the number of speculative traders which can be supported in a zero profit equilibrium and in a maximum profit equilibrium, as in section 3.

Proposition 6 The per speculator percentage return on information remains constant and bounded away from zero when:

1. The variance $V_{\alpha}$ of the value of the risky security increases;

2. The variance $V_{L} V_{\alpha}$ of the number of liquidity traders increases;

3. The cost $c$ of information tends to zero.

Proposition 6 shows that our result is robust in the following sense: while the equilibrium number of traders may change in response to parameter variation, the net per-speculator percentage profits remain bounded away from zero. This shows that positive profits from publicly available information is not a fragile feature of our model.

Corollary 7 The expected per-speculator percentage profits are proportional to

$$
E\{\sqrt{\tilde{m}}\} E\left\{\frac{1}{\sqrt{1+\tilde{m}}}\right\}
$$

If the distribution of the number of noise traders changes then the effect upon the equilibrium per-speculator profit is determined by the change in $E\{\sqrt{\tilde{m}}\} E\left\{\frac{1}{\sqrt{1+\tilde{m}}}\right\}$ : if $E\{\sqrt{\tilde{m}}\}$ grows faster than $E\left\{\frac{1}{\sqrt{1+\tilde{m}}}\right\}$ decreases then profits will increase; they will otherwise decrease. When $\tilde{m}$ is drawn from a Poisson distribution, numerical simulations suggest that as the mean increases, so does the per-speculator percentage profit.

Proposition 8 The expression $\left(s^{Z P}-s^{M P}\right)$ is monotonically decreasing in $c$. It follows that, modulo integer effects, $k$ is decreasing in $c$.

Proposition 8 is illustrated in figure 2. It states that as the cost $c$ of information acquisition tends to zero, the difference between the maximum (zero per trader profit) number $n^{Z P}$ of informed speculators which can be sustained in equilibrium and the minimum (maximum per trader profit) number $n^{M P}$ increases. 


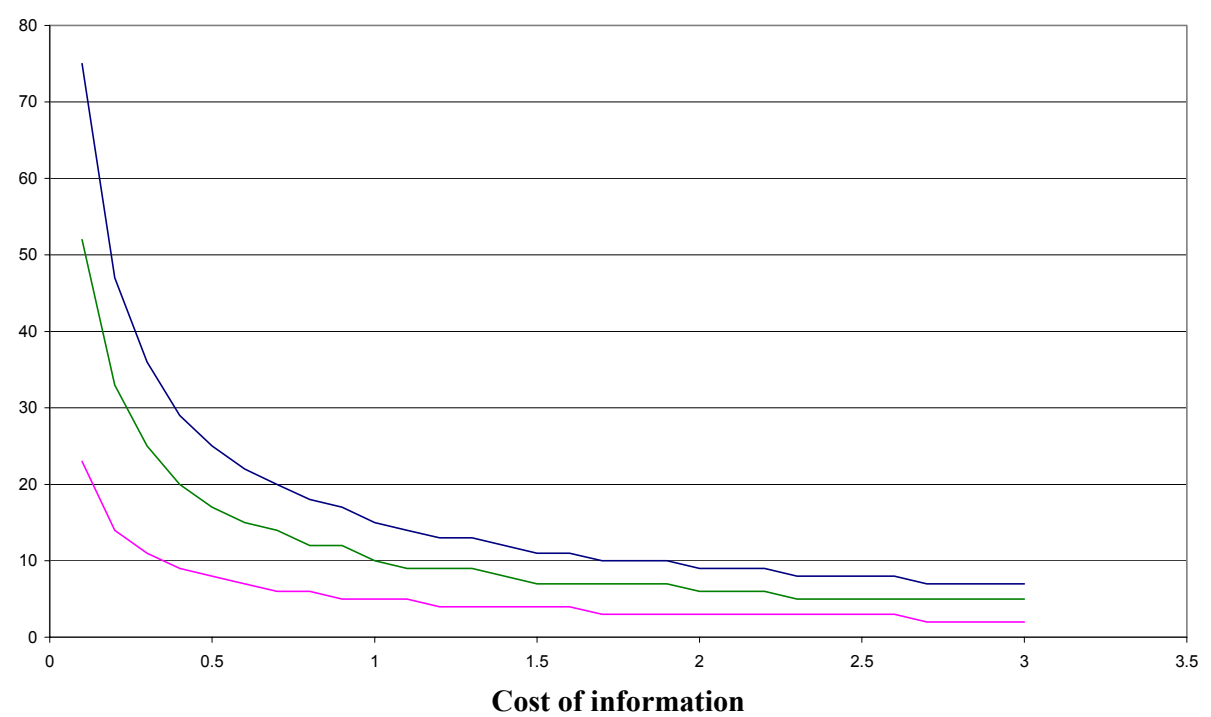

Figure 2: The graph shows the effect of varying the cost $c$ of information when $E\{\sqrt{\tilde{m}}\}=3$, $E\left\{\frac{1}{\sqrt{1+\tilde{m}}}\right\}=\frac{1}{2}$ and $V_{\alpha}=V_{L}=10$. The highest plot is of $n^{Z P}$, the middle one is of $n^{M P}$ and the lowest is $n^{Z P}-n^{M P}$.

Propositions 6 and 8 illustrate an important and counter-intuitive feature of our model. In a standard model with costless information acquisition and free entry the per-speculator profit would be zero. When the model is extended to allow for the relationship between information acquisition and expectations we have shown that for any cost of information acquisition $c$, rational expectations equilibria exist with limited entry and positive expected percentage profits.

\section{Conclusion}

The traditional market microstructure literature has assumed that free speculator entry drives profits down to zero. This assumption neglects the possible strategic effect that the information acquisition decision of one trader might have upon others. In this paper we show that this effect matters. Beliefs can serve as a barrier to entry which supports positive speculator profits with free entry. This is consistent with practitioner explanations of supernormal profits.

It is an important goal of normative finance to explain real market phenomena within the rational framework. In the real world, speculators make supernormal profits. This paper provides a rational framework where this is both possible and plausible. 
Appendix

Proposition 9 proves the existence and uniqueness of equilibrium when $m$ and $n$ are common knowledge. It is required to prove the results in the text.

Proposition 9 Suppose that the respective numbers $m$ and $n$ of liquidity traders and speculators are common knowledge. Then there exists a unique linear Nash equilibrium. In equilibrium the expected profit excluding information costs $P_{n, m}$ which informed traders earn is as follows:

$$
P_{n, m}=\frac{V_{\alpha}}{n+1} \sqrt{\frac{m V_{L}}{2 n}} .
$$

Proof. Suppose that $m$ and $n$ are common knowledge and that every informed trader except the $k$ th follows strategies given by parts $2(\mathrm{a})$ and 2(b) of definition 1 . Given a signal $\alpha+\varepsilon \sqrt{V_{\alpha}}$, the expected profit which the $k$ th trader will earn from a position $x_{k}$ is

$$
\begin{aligned}
& E\left\{x_{k}(\tilde{S}-\tilde{P}(\tilde{\omega})) \mid \alpha+\varepsilon \sqrt{V_{\alpha}}\right\} \\
& =E\left\{x_{k} \tilde{\alpha}-\lambda x_{k}^{2}-\lambda x_{k} \sum_{\substack{i=1 \\
i \neq k}}^{n} \tilde{x}_{i} \beta\left(\tilde{\alpha}+\tilde{\varepsilon} \sqrt{V_{\alpha}}\right)-\lambda x_{k} \sum_{j=1}^{m} \tilde{y}_{j} \mid \alpha+\varepsilon \sqrt{V_{\alpha}}\right\} \\
& =\frac{x_{k}}{2}\left(\alpha+\varepsilon \sqrt{V_{\alpha}}\right)-\lambda x_{k}^{2}-\lambda x_{k}(n-1) \beta\left(\alpha+\varepsilon \sqrt{V_{\alpha}}\right) .
\end{aligned}
$$

The $k$ th informed trader will select $x_{k}$ so as to maximize this expression. Differentiation immediately yields

$$
x_{k}=\frac{1}{2 \lambda}\left(\alpha+\varepsilon \sqrt{V_{\alpha}}\right)\left(\frac{1}{2}-\beta(n-1) \lambda\right),
$$

and this expression equals $\beta\left(\alpha+\varepsilon \sqrt{V_{\alpha}}\right)$ in equilibrium, so that

$$
\beta=\frac{1}{2 \lambda(n+1)} .
$$

Inserting equation 9 into the objective function equation 8 yields the following:

$$
\begin{aligned}
& \left(\frac{\beta}{2} \frac{2}{n+1}-\lambda \beta^{2}\right)\left(\alpha+\varepsilon \sqrt{V_{\alpha}}\right)^{2} \\
& =\frac{1}{4 \lambda(1+n)^{2}}\left(\alpha+\varepsilon \sqrt{V_{\alpha}}\right)^{2} .
\end{aligned}
$$

Take expectations with respect to $\alpha$ and $\varepsilon$ to determine the trader's expected profit:

$$
\frac{V_{\alpha}}{2 \lambda(n+1)^{2}} \text {. }
$$


Part 1(a) of definition 1 and the projection theorem imply that

$$
P(\tilde{\omega})=\bar{S}+\lambda \tilde{\omega}=E\{\tilde{S} \mid \tilde{\omega}\}=\bar{S}+\frac{\operatorname{cov}(\tilde{S}, \tilde{\omega})}{\operatorname{var}(\tilde{\omega})} \tilde{\omega},
$$

whence

$$
\lambda=\frac{\operatorname{cov}(\tilde{S}, \tilde{\omega})}{\operatorname{var}(\tilde{\omega})} .
$$

The equilibrium value for $\tilde{\omega}$ is $n \beta\left(\tilde{\alpha}+\tilde{\varepsilon} \sqrt{V_{\alpha}}\right)+\sum_{j=1}^{m} \tilde{y}_{j}$, so

$$
\begin{aligned}
\operatorname{cov}(\tilde{S}, \tilde{\omega}) & =\operatorname{cov}(\tilde{\alpha}, \tilde{\omega})=\beta n V_{\alpha} \\
\operatorname{var}(\tilde{\omega}) & =2 n^{2} \beta^{2} V_{\alpha}+m V_{L} V_{\alpha} \\
& =V_{\alpha}\left(2 n^{2} \beta^{2}+m V_{L}\right)
\end{aligned}
$$

Substituting equations 13 and 14 into equation 12 and solving for $\lambda$ yields equation 15 .

$$
\lambda_{n, m}=\frac{1}{n+1} \sqrt{\frac{n}{2 m V_{L}}} .
$$

Inserting this expression back into equation 11 yields equation 7 , as required.

\section{Proof of Lemma 2}

Conditional upon a signal $\alpha+\varepsilon \sqrt{V_{\alpha}}$ the expected profit which the $(N+1)$ th speculator will make from buying $\bar{x}$ shares is

$$
\begin{aligned}
& E\left\{\bar{x}(\tilde{S}-\tilde{P}(\tilde{\omega})) \mid \alpha+\varepsilon \sqrt{V_{\alpha}}\right\} \\
& =E\left\{\bar{x} \tilde{\alpha}-\lambda \bar{x}^{2}-\lambda \bar{x} \sum_{i=1}^{n} \beta_{n, \tilde{m}+1}\left(\tilde{\alpha}+\tilde{\varepsilon} \sqrt{V_{\alpha}}\right)-\lambda \bar{x} \sum_{j=1}^{\tilde{m}} \tilde{y}_{j} \mid \alpha+\varepsilon \sqrt{V_{\alpha}}\right\} \\
& =E_{\tilde{m}}\left\{\bar{x}\left(\frac{1}{2}-\beta_{n, \tilde{m}+1} \lambda_{n, \tilde{m}+1} n\right)\left(\alpha+\varepsilon \sqrt{V_{\alpha}}\right)-\lambda_{n, \tilde{m}+1} \bar{x}^{2}\right\} \\
& =\frac{\bar{x}}{2} \frac{1}{n+1}\left(\alpha+\varepsilon \sqrt{V_{\alpha}}\right)-\frac{\bar{x}^{2}}{n+1} \sqrt{\frac{n}{2 V_{L}}} E\left\{\frac{1}{\sqrt{\tilde{m}+1}}\right\},
\end{aligned}
$$

The first order condition then implies that

$$
\bar{x}=\frac{1}{2 E\left\{\frac{1}{\sqrt{\tilde{m}+1}}\right\}} \sqrt{\frac{V_{L}}{2 n}}\left(\alpha+\varepsilon \sqrt{V_{\alpha}}\right) .
$$

Substituting this expression back into equation 16 gives us the following expression for the conditional profit of the new entrant:

$$
\frac{1}{8 E\left\{\frac{1}{\sqrt{\tilde{m}+1}}\right\}(n+1)} \sqrt{\frac{V_{L}}{2 n}}\left(\alpha+\varepsilon \sqrt{V_{\alpha}}\right)^{2} .
$$


Taking expectations of this expression with respect to $\alpha$ and $\varepsilon$ gives us equation 1 , as required.

Proof of Lemma 3

Equation 3is obtained by taking the expectation of equation 7 with respect to $m$.

Proof of Proposition 4

$P^{\prime}(n)<0$ so to prove the first part of the proposition we need only prove that $P(n+1)>\Pi(n)$.

This is true if and only if

$$
4 E\left\{\frac{1}{\sqrt{1+\tilde{m}}}\right\} E\{\sqrt{\tilde{m}}\}>\sqrt{\frac{n+1}{n}} \frac{n+2}{n+1} .
$$

Note that the right hand side of this expression is less than or equal to $\frac{3}{\sqrt{2}}$. It therefore suffices to show that $4 E\left\{\frac{1}{\sqrt{1+\tilde{m}}}\right\} E\{\sqrt{\tilde{m}}\}>\frac{3}{\sqrt{2}}$. Note that $\sqrt{\tilde{m}}$ and $\frac{1}{\sqrt{1+\tilde{m}}}$ are negatively correlated, so that $E\left\{\sqrt{\frac{\tilde{m}}{1+\tilde{m}}}\right\}-\left\{\frac{1}{\sqrt{1+\tilde{m}}}\right\} E\{\sqrt{\tilde{m}}\}<0$. Since $\sqrt{\frac{\tilde{m}}{1+\tilde{m}}}$ is increasing in $\tilde{m}$, it follows immediately that the right hand side of equation 17 is greater than $\frac{4}{\sqrt{2}}$, which exceeds $\frac{3}{\sqrt{2}}$ as required. This proves the first part of the proposition.

The second part of the proposition is a trivial consequence of lemmas 3 and 3 .

\section{Proof of Proposition 6}

Equations 1 and 2 imply that

$$
\Pi(n)=P(n) \cdot \frac{1}{4 E\{\sqrt{\tilde{m}}\} E\left\{\frac{1}{\sqrt{1+\tilde{m}}}\right\}} .
$$

Recall from section 3 that by definition $P\left(s^{Z P}\right)=c$ and $\Pi\left(s^{M P}\right)=c$. It follows that

$$
\begin{aligned}
\frac{P\left(s^{M P}\right)-c}{c} & =\frac{4 E\{\sqrt{\tilde{m}}\} E\left\{\frac{1}{\sqrt{1+\tilde{m}}}\right\}}{c} \Pi\left(s^{M P}\right)-1 \\
& =4 E\{\sqrt{\tilde{m}}\} E\left\{\frac{1}{\sqrt{1+\tilde{m}}}\right\}-1,
\end{aligned}
$$

from which proposition 6 follows immediately. 


\section{Proof of Proposition 8}

Implicit differentiation of equation 5 with respect to $c$ yields the following:

$$
\frac{\partial s^{Z P}}{\partial c}=-\frac{2 s^{Z P}\left(s^{Z P}+1\right)^{2}}{3 s^{Z P}+1} \sqrt{\frac{2 s^{Z P}}{V_{L}}} \frac{1}{V_{\alpha} E\{\sqrt{\tilde{m}}\}} .
$$

Similarly, the following expression is obtained by differentiating equation 6 :

$$
\frac{\partial s^{M P}}{\partial c}=-\frac{2 s^{M P}\left(s^{M P}+1\right)^{2}}{3 s^{M P}+1} \sqrt{\frac{2 s^{M P}}{V_{L}}} \frac{4 E\left\{\frac{1}{\sqrt{1+\tilde{m}}}\right\}}{V_{\alpha}} .
$$

Equations 19 and 20 imply the following:

$$
\begin{aligned}
& \frac{\partial}{\partial c}\left(s^{Z P}-s^{M P}\right) \\
& =\frac{2}{V_{\alpha}} \sqrt{\frac{2}{V_{L}}}\left\{\frac{s^{M P} \sqrt{s^{M P}}\left(s^{M P}+1\right)^{2}}{3 s^{M P}+1} 4 E\left\{\frac{1}{\sqrt{1+\tilde{m}}}\right\}-\frac{s^{Z P} \sqrt{s^{Z P}}\left(s^{Z P}+1\right)^{2}}{3 s^{Z P}+1} \frac{1}{E\{\sqrt{\tilde{m}}\}}\right\} \\
& <0 \text { iff } 4 E\{\sqrt{\tilde{m}}\} E\left\{\frac{1}{\sqrt{1+\tilde{m}}}\right\}<\frac{s^{Z P}\left(s^{Z P}+1\right)}{s^{M P}\left(s^{M P}+1\right)} \frac{3 s^{Z P}+1}{3 s^{M P}+1} \sqrt{\frac{s^{Z P}}{s^{M P}}} .
\end{aligned}
$$

Substituting from equations 6 and 5 respectively for $s^{M P}+1$ and $s^{Z P}+1$ yields the following equivalent condition:

$$
\frac{3 s^{M P}+1}{s^{M P}}>\frac{3 s^{Z P}+1}{s^{Z P}} .
$$

This reduces to $s^{Z P}>s^{M P}$, which is true by lemma 5 .

\section{REFERENCES}

Admati, A. R. and P. Pfleiderer (1988), 'A Theory of Intraday Patterns: Volume and Price Variability', Review of Financial Studies, Vol. 1, No. 1 (Spring), pp. 3-40.

Foster, F. D. and S. Viswanathan (1993), 'The Effect of Public Information and Competition on Trading Volume and Price Volatility', Review of Financial Studies, Vol. 6, No. 1, pp. 23 - 56.

Grossman, S. J. and J. E. Stiglitz (1980), 'On the Impossibility of Informationally Efficient Markets', American Economic Review, Vol. 70, No. 3 (June), pp. 393-403.

Kreps, D. M. and R. Wilson (1982), 'Sequential Equilibria', Econometrica, Vol. 50, No. 4 (July), pp. $863-894$.

Kyle, A. S. (1985), 'Continuous Auctions and Insider Trading', Econometrica, Vol. 53, No. 6 (November), pp. 1315-1335. 
— (1989), 'Informed Speculation with Imperfect Competition', Review of Economic Studies, Vol. 56, No. 3 (July), pp. $317-356$.

Morrison, A. D. (2003), 'Competition and Information Production in Market Maker Models', Journal of Business Finance and Accounting (Forthcoming).

Schwager, J. D. (1989), Market Wizards: Interviews with Top Traders, New York Institute of Finance, New York, NY.

Spiegel, M. and A. Subrahmanyam (1992), 'Informed Speculation and Hedging in a Noncompetitive Securities Market', Review of Financial Studies, Vol. 5, No. 2, pp. 307 - 329.

Verrecchia, R. E. (1982), 'Information Acquisition in a Noisy Rational Expectations Economy', Econometrica, Vol. 50, No. 6 (November), pp. 1415 - 1430. 